\title{
Identification of Putative Early Atherosclerosis Biomarkers by Unsupervised Deconvolution of Heterogeneous Vascular Proteomes
}

Sarah J Parker ${ }^{1}$, Lulu Chen², Weston Spivia ${ }^{1}$, Georgia Saylor ${ }^{3}$, Chunhong Mao ${ }^{4}$, Vidya Venkatraman ${ }^{1}$, Ronald J. Holewinski ${ }^{1}$, Mitra Mastali ${ }^{1}$, Rakhi Pandey ${ }^{1}$, Grace Athas ${ }^{5}$, Guoqiang Yu², Qin Fu ${ }^{1}$, Dana Troxlair ${ }^{5}$, Richard Vander Heide ${ }^{5}$, David Herrington ${ }^{3 *}$, Jennifer E. Van Eyk ${ }^{1 *}$, and Yue Wang ${ }^{2 *}$

* equal contribution

\section{Author Affiliations:}

1. Cedars Sinai Medical Center, Heart Institute \& Advanced Clinical Biosystems Research Institute, Los Angeles, CA

2. Department of Electrical and Computer Engineering, Virginia Polytechnic Institute and State University, Arlington, VA

3. Wake Forest University, Department of Cardiovascular Medicine, Winston-Salem, NC

4. Biocomplexity Institute \& Initiative, University of Virginia, Charlottesville, VA

5. Louisiana State University, Department of Pathology, New Orleans, LA

\section{Corresponding Author:}

Sarah J Parker

Smidt Heart Institute

Cedars Sinai Medical Center

Advanced Health Sciences Pavilion A9228

8700 Beverly Boulevard

Los Angeles CA 90048

423-431-2384

sarah.parker@cshs.org

Table of Contents - Supplementary Files

Supplementary Text - pg. S2-S4

Supplementary Figure 1 - pg S5

Supplementary Figure 2 - pg S6

Supplementary Figure 3 - pg S7 (caption pg S8)

References to Supplementary Text - pg S8-9 


\section{Supplemental Text}

\section{Functional Description and known CAD-Associations of the 10 Elastic Net selected Biomarker Candidates}

APOM. Apolipoprotein M is a known lipoprotein component in HDL cholesterol and has shown antiatherogenic properties in mouse studies. In previous work, it was not found to be a significant predictor of CAD risk, however these results were observed in a cohort that was predominantly male ( $>70 \%$ male), which may explain discrepancies with our entirely female cohort. ${ }^{1-3}$

PLMN: Plasminogen is the circulating precursor to plasmin, which, upon activation by tissue derived proteases mediates localized inflammatory responses, complement activation, and macrophage recruitment. Recently, elevated plasminogen was reported among patients with stable CAD relative to those experiencing acute thrombotic myocardial infarction ${ }^{4}$. Interestingly, in another recent study of circulating CVD risk biomarker panels, PLMN was shown to be lower in individuals who had a CV event relative to normal ${ }^{5}$ when measured in depleted plasma but subsequently elevated in higher CV risk cohort in undepleted plasma, consistent with our results.

A2MG: Alpha-2 Macroglobulin is a large protein that exists in multimeric form with a major function as a negative regulator of proteinases. It is also known to bind and sequester/inhibit many other proteins including cytokines such as IL1B and fibroblast growth factor ${ }^{6}$. A2MG has been shown to be associated with select aspects of atherosclerotic plaques upon imaging ultrasound, namely more intense calcification and necrotic tissue ${ }^{7}$, though overall it demonstrated low strength as a circulating biomarker in that study.

IGHA2: Constant region of immunoglobulin heavy chain. Immunoglobulins are secreted by b lymphocytes and mediate humoral immunity. Elevated IGHA2 has been identified as a potential biomarker of renal function during hypertension ${ }^{5}$ as well as a component of a putative panel for early detection of heart failure . $^{8}$ APOC2: Apolipoprotein C-II is a member of a cluster of apolipoproteins that circulate with very low density lipoproteins, and regulate triglyceride metabolism via lipoprotein lipase activation ${ }^{9}$. Inactivating mutations and 
APOC2 deficiency are associated with hypertriglyceremia and atherosclerosis, thus it is unclear whether elevation of this apolipoprotein in lesions and circulating plasma of CAD patients is an attempt to compensate or is pro-atherogenic.

VTNC: Vitronectin is localized to vascular extracellular matrix protein and has pleiotropic functions including regulation of proteolysis, complement activation, and cellular migration ${ }^{10}$. It has been shown to be upregulated in many studies of circulating biomarkers of atherosclerosis ${ }^{11,12}$ potentially independent of circulating lipid levels ${ }^{13}$.

CD14: Cluster of differentiation 14 is cell surface protein associated with Toll like Receptor signaling. It is a marker of macrophages, and as such has been associated with atherosclerotic lesions previously ${ }^{14}$. Interestingly, soluble forms of CD14 exist separate from the cell surface associated protein, and while macrophage-associated CD14 may be expected to increase in atherosclerotic lesions due to inflammation and the role of macrophages in pathogenesis, evidence for soluble CD14 as a marker of atherosclerosis has been mixed but some forms have been shown to decrease in patients with CAD.

ITIH2: Inter-alpha trypsin inhibitor heavy chain 2 is one of the non-enzymatic components of the inter-alpha trypsin inhibitor complex. This complex is both a serum protease inhibitor of chymotrypsin, leukocyte elastase, and plasmin as well as a stabilizer of the extracellular matrix in tissue via interactions with hyaluron ${ }^{15}$. Reduced ITIH2 was recently reported among postmenopausal women with CAD, a cohort very similar to that reported in the current study ${ }^{16}$.

TSP2: Thrombospondin-2 is a matricellular protein that regulates proteases and growth factor availability and activity in the extracellular environment ${ }^{17}$. Among patients with established CHD, elevated TSP2 levels are associated with mortality ${ }^{18}$. Higher levels of TSP2 have also been associated with greater CVD risk in a 'general population'19. Interestingly, we observed generally lower TSP2 among women with CAD in our study despite high tissue expression in fibrous plaque lesions. Previous studies have used serum and not plasma, and 
previously published studies have used ELISA as opposed to mass spectrometry based quantification used here.

URP2: This protein is named Fermitin family homolog 3 or Kindlin-3 and is found in atherosclerotic plaques, particularly unstable plaques, but tends to colocalize with $\mathrm{M} 2$ 'anti-inflammatory' macrophages ${ }^{20}$. Despite upregulation in lesion tissue, this protein was slightly negatively associated with CAD which was consistent with another recent report on plasma biomarkers of CAD in post-menopausal women ${ }^{16}$. 
MDL Curve

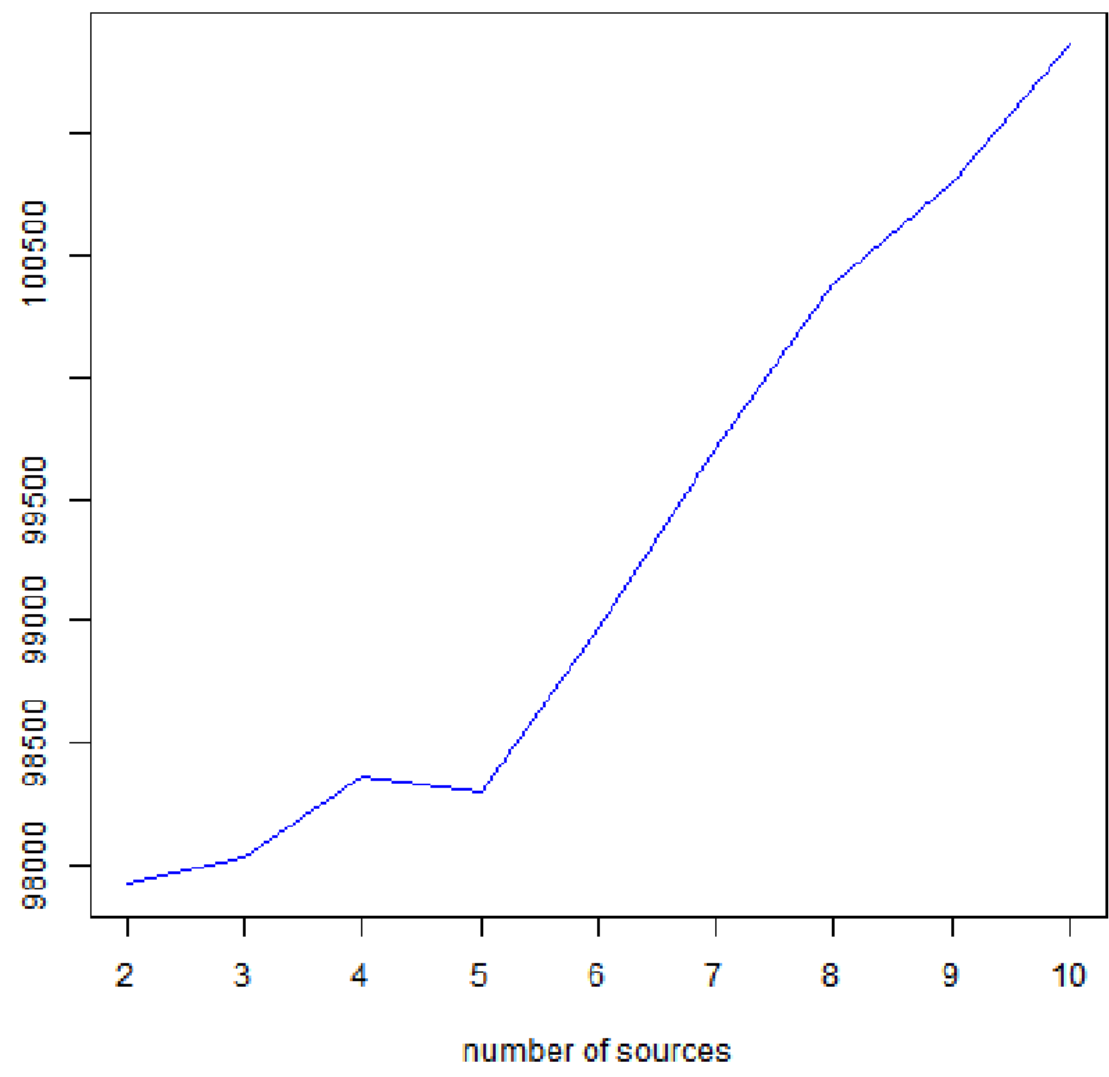

MDL Curve

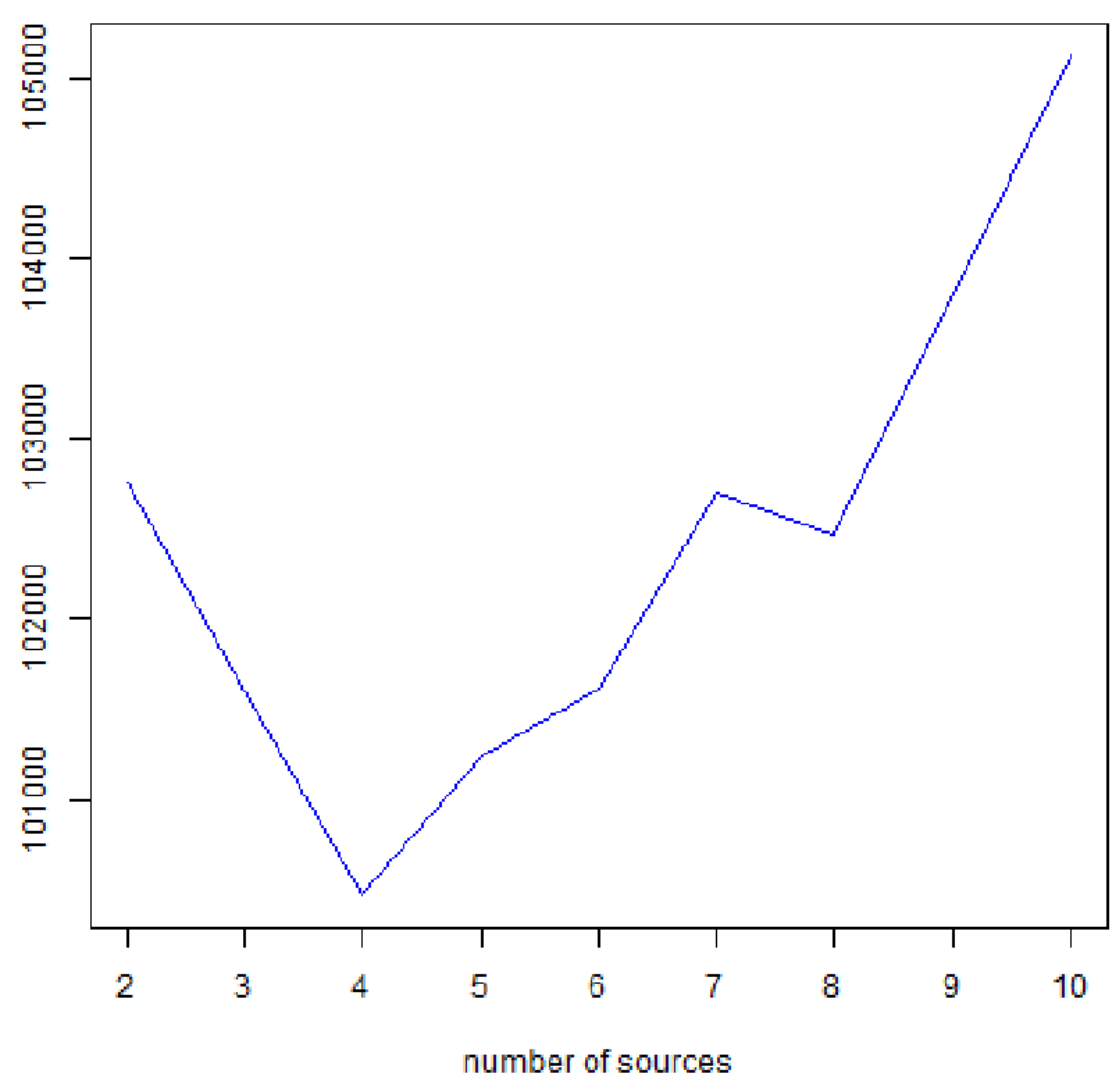

Supplementary Figure 1. Minimum Description Length (MDL) Plots generated during CAM Analysis. The CAM generated MDL curves for the AA (left) and LAD (right) show minima occurring at 2 sources for the AA and 4 sources for the LAD. 


\section{Discovery AA Samples}

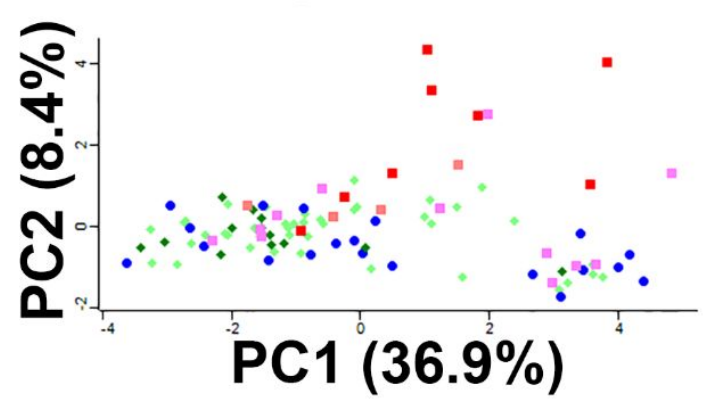

\section{Discovery LAD Samples}

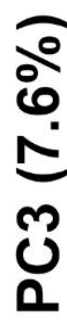

仓̊̊

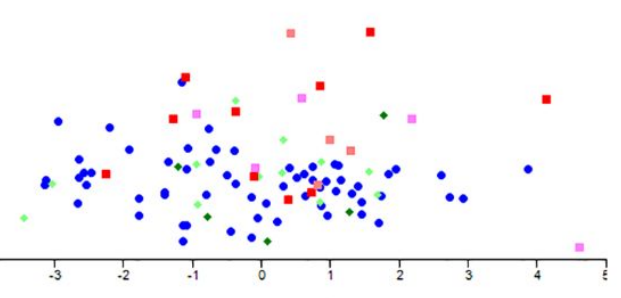

PC1 (20.2\%) $\geq 50 \%$ Fibrous Plaque

$1-49 \%$ Fibrous Plaque

Mixed Fatty Streak \& Fibrous Plaque

$\geq \mathbf{5 0} \%$ Fatty Streak

$1-49 \%$ Fatty Streak

Normal (No lesion)

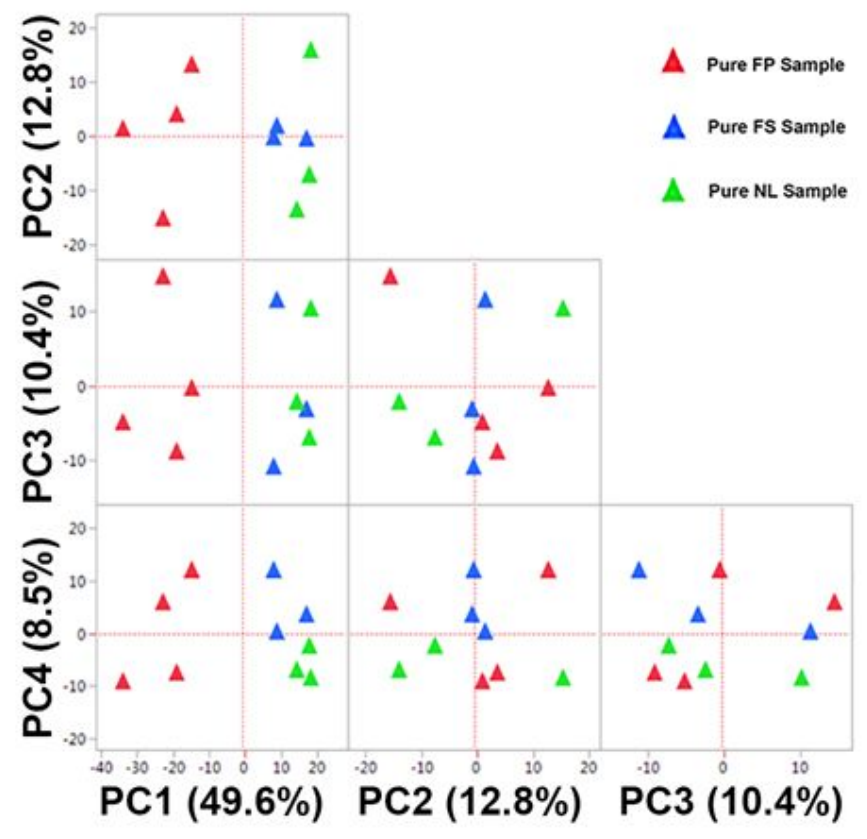

Supplemental Figure 2. Principal Component Analysis of heterogenous discovery samples (left panel A) and Pure Lesion / normal validation samples (right panel B).

A. Principal component analysis showed some separation of fibrous plaque samples along PC2 (AA) or PC3 (LAD), however separation was imperfect and the continuous nature of lesion burden made categorical classification of tissues difficult, supporting alternative analysis methods for these data. B. Analysis of the DIA-MS dataset from pure lesion samples demonstrates that when pathology is straight forward (e.g., 'categorical' or 'case-control') the fibrous plaque (FP) lesions (red triangles) can be clearly separated from Normal (NL, green triangles) and Fatty Streak (FS, blue triangles) along principal component (PC) 1. Additional separation between NL and FS can be achieved along PC4. Thus, a plot of PC1 versus PC4 (lower left corner) best segregates all groups. 
A
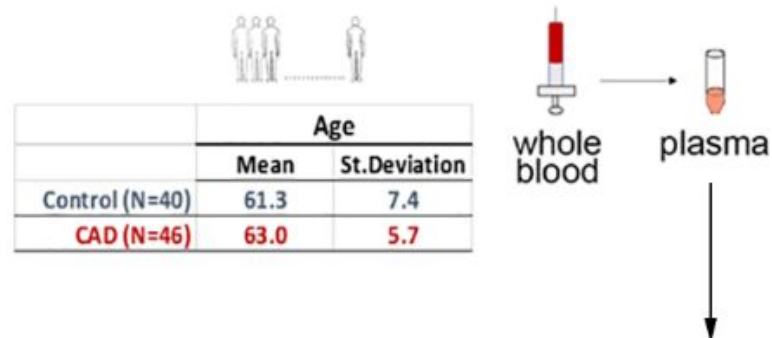

Multiple Reaction Monitoring Mass Spectrometry (Scheduled 42 protein plex)
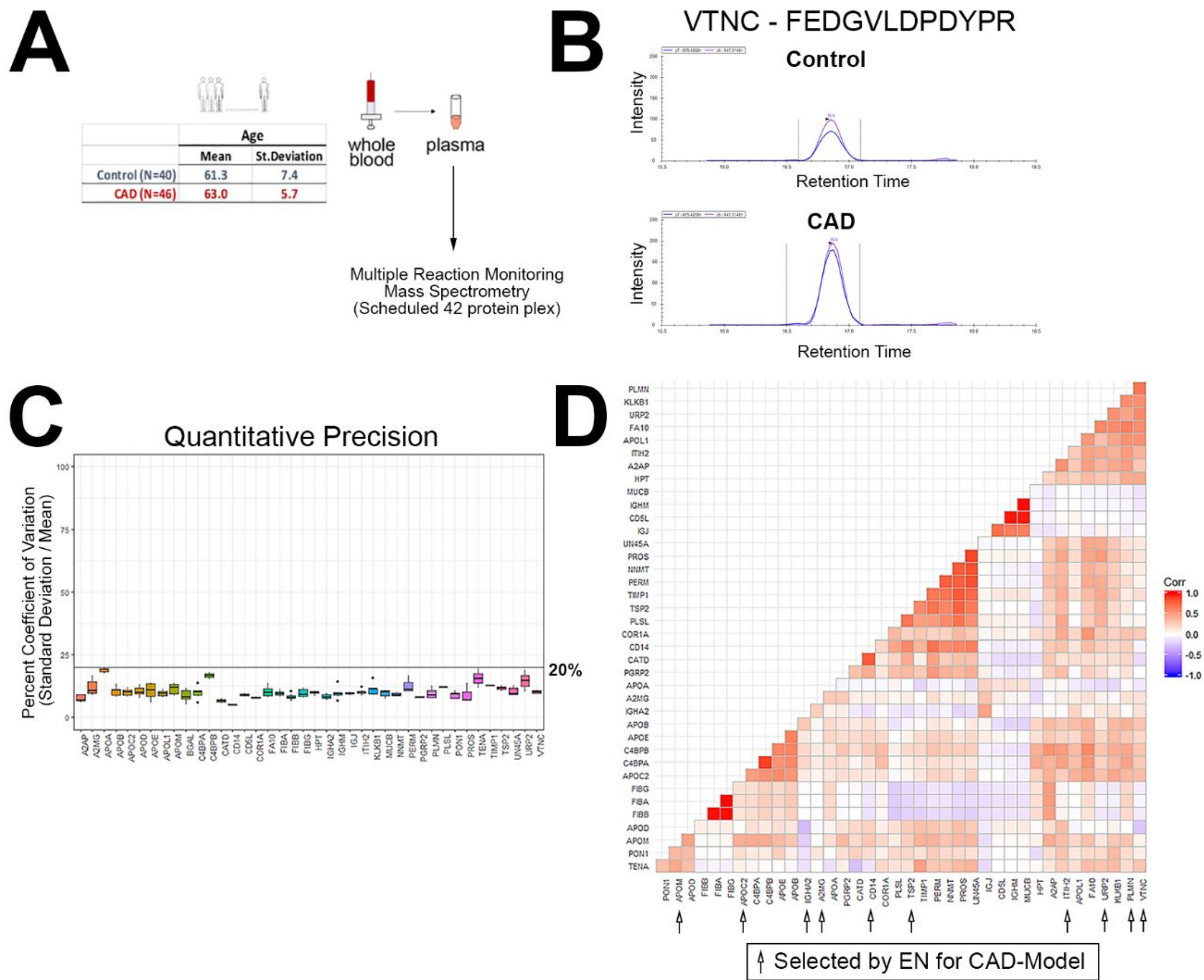
Supplementary Figure 3. Initial testing and validation of a preliminary FP-marker panel in human plasma. (A) Patient characteristics and workflow schematic of the test cohort for plasma FP marker validation. (B) Example peptide quantification result for the protein vitronectin in a representative control and CAD patient plasma sample. (C) Distribution of fragment coefficient of variation values for each protein across all of the quality control pooled plasma samples acquired (D) matrix of pearson correlation coefficient strength, presented as a heatmap, between each of the 39 quantifiable proteins in the human plasma cohort. Arrows indicate candidates that were ultimately selected as predictors by Elastic Net in the logistic regression model.

\section{References}

1. Ahnstrom J, Axler O, Jauhiainen M, Salomaa V, Havulinna AS, Ehnholm C, Frikke-Schmidt R, TybjaergHansen A, Dahlback B. Levels of apolipoprotein $M$ are not associated with the risk of coronary heart disease in two independent case-control studies. J Lipid Res 2008;49(9):1912-7.

2. Arkensteijn BW, Berbee JF, Rensen PC, Nielsen LB, Christoffersen C. The apolipoprotein m-sphingosine1-phosphate axis: biological relevance in lipoprotein metabolism, lipid disorders and atherosclerosis. Int J Mol Sci 2013;14(3):4419-31.

3. Christoffersen C, Benn M, Christensen PM, Gordts PL, Roebroek AJ, Frikke-Schmidt R, Tybjaerg-Hansen $A$, Dahlback B, Nielsen LB. The plasma concentration of HDL-associated apoM is influenced by LDL receptormediated clearance of apoB-containing particles. J Lipid Res 2012;53(10):2198-204.

4. DeFilippis AP, Chernyavskiy I, Amraotkar AR, Trainor PJ, Kothari S, Ismail I, Hargis CW, Korley FK, Leibundgut G, Tsimikas S, Rai SN, Bhatnagar A. Circulating levels of plasminogen and oxidized phospholipids bound to plasminogen distinguish between atherothrombotic and non-atherothrombotic myocardial infarction. J Thromb Thrombolysis 2016;42(1):61-76.

5. Baldan-Martin M, Lopez JA, Corbacho-Alonso N, Martinez PJ, Rodriguez-Sanchez E, Mourino-Alvarez L, Sastre-Oliva T, Martin-Rojas T, Rincon R, Calvo E, Vazquez J, Vivanco F, Padial LR, Alvarez-Llamas G, RuizHurtado G, Ruilope LM, Barderas MG. Potential role of new molecular plasma signatures on cardiovascular risk stratification in asymptomatic individuals. Sci Rep 2018;8(1):4802.

6. Rehman AA, Ahsan H, Khan FH. alpha-2-Macroglobulin: a physiological guardian. J Cell Physiol 2013;228(8):1665-75.

7. Battes LC, Akkerhuis KM, Cheng JM, Garcia-Garcia HM, Oemrawsingh RM, de Boer SP, Regar E, van Geuns RJ, Serruys PW, Boersma E, Kardys I. Circulating acute phase proteins in relation to extent and composition of coronary atherosclerosis and cardiovascular outcome: results from the ATHEROREMO-IVUS study. Int J Cardiol 2014;177(3):847-53.

8. Zhang X, Walsh T, Atherton JJ, Kostner K, Schulz B, Punyadeera C. Identification and Validation of a Salivary Protein Panel to Detect Heart Failure Early. Theranostics 2017;7(18):4350-4358.

9. Wolska A, Dunbar RL, Freeman LA, Ueda M, Amar MJ, Sviridov DO, Remaley AT. Apolipoprotein C-II: New findings related to genetics, biochemistry, and role in triglyceride metabolism. Atherosclerosis

2017;267:49-60.

10. Schvartz I, Seger D, Shaltiel S. Vitronectin. Int J Biochem Cell Biol 1999;31(5):539-44.

11. Lepedda AJ, Lobina O, Rocchiccioli S, Nieddu G, Ucciferri N, De Muro P, Idini M, Nguyen HQ, Guarino A, Spirito R, Formato M. Identification of differentially expressed plasma proteins in atherosclerotic patients with type 2 diabetes. J Diabetes Complications 2016;30(5):880-6. 
12. Yaghoubi A, Ghojazadeh M, Abolhasani S, Alikhah H, Khaki-Khatibi F. Correlation of Serum Levels of Vitronectin, Malondialdehyde and Hs- CRP With Disease Severity in Coronary Artery Disease. J Cardiovasc Thorac Res 2015;7(3):113-7.

13. Ekmekci H, Sonmez H, Ekmekci OB, Ozturk Z, Domanic N, Kokoglu E. Plasma vitronectin levels in patients with coronary atherosclerosis are increased and correlate with extent of disease. J Thromb Thrombolysis 2002;14(3):221-5.

14. An D, Hao F, Zhang F, Kong W, Chun J, Xu X, Cui MZ. CD14 is a key mediator of both lysophosphatidic acid and lipopolysaccharide induction of foam cell formation. J Biol Chem 2017;292(35):14391-14400.

15. Bost F, Diarra-Mehrpour M, Martin JP. Inter-alpha-trypsin inhibitor proteoglycan family--a group of proteins binding and stabilizing the extracellular matrix. Eur J Biochem 1998;252(3):339-46.

16. Prentice RL, Paczesny S, Aragaki A, Amon LM, Chen L, Pitteri SJ, McIntosh M, Wang P, Buson Busald T, Hsia J, Jackson RD, Rossouw JE, Manson JE, Johnson K, Eaton C, Hanash SM. Novel proteins associated with risk for coronary heart disease or stroke among postmenopausal women identified by in-depth plasma proteome profiling. Genome Med 2010;2(7):48.

17. Bornstein P, Armstrong LC, Hankenson KD, Kyriakides TR, Yang Z. Thrombospondin 2, a matricellular protein with diverse functions. Matrix Biol 2000;19(7):557-68.

18. Berezin AE, Kremzer AA, Samura TA. Circulating thrombospondin-2 in patients with moderate-tosevere chronic heart failure due to coronary artery disease. Journal of Biomedical Research 2016;30(1):32-39.

19. Morikawa N, Adachi H, Enomoto M, Fukami A, Kumagai E, Nakamura S, Nohara Y, Nakao E, Kono S, Tsuru T, Sakaue A, Hamamura H, Fukumoto Y. Thrombospondin-2 as a Potential Risk Factor in a General Population. Int Heart J 2019.

20. Oksala N, Parssinen J, Seppala I, Klopp N, Illig T, Laaksonen R, Levula M, Raitoharju E, Kholova I, Sioris T, Kahonen M, Lehtimaki T, Hytonen VP. Kindlin 3 (FERMT3) is associated with unstable atherosclerotic plaques, anti-inflammatory type II macrophages and upregulation of beta-2 integrins in all major arterial beds.

Atherosclerosis 2015;242(1):145-54. 